\title{
Confocal fluorescence microscopy for rapid evaluation of invasive tumor cellularity of inflammatory breast carcinoma core needle biopsies
}

\author{
Jessica Dobbs • Savitri Krishnamurthy • \\ Matthew Kyrish • Ana Paula Benveniste • \\ Wei Yang • Rebecca Richards-Kortum
}

Received: 3 July 2014/ Accepted: 24 October 2014/Published online: 23 November 2014

(C) The Author(s) 2014. This article is published with open access at Springerlink.com

\begin{abstract}
Tissue sampling is a problematic issue for inflammatory breast carcinoma, and immediate evaluation following core needle biopsy is needed to evaluate specimen adequacy. We sought to determine if confocal fluorescence microscopy provides sufficient resolution to evaluate specimen adequacy by comparing invasive tumor cellularity estimated from standard histologic images to invasive tumor cellularity estimated from confocal images of breast core needle biopsy specimens. Grayscale confocal fluorescence images of breast core needle biopsy specimens were acquired following proflavine application. A breast-dedicated pathologist evaluated invasive tumor cellularity in histologic images with hematoxylin and eosin staining and in grayscale and false-colored confocal images of cores. Agreement between cellularity estimates was quantified using a kappa coefficient. 23 cores from 23 patients with suspected inflammatory breast carcinoma were imaged. Confocal images were acquired in an average
\end{abstract}

J. Dobbs · M. Kyrish · R. Richards-Kortum $(\varangle)$

Department of Bioengineering, Rice University, 6500 Main

Street, BRC 502, Houston, TX 77030, USA

e-mail: rkortum@rice.edu

S. Krishnamurthy

Department of Pathology, University of Texas MD Anderson Cancer Center, 1515 Holcombe Boulevard, Unit 1350, Houston, TX 77030, USA

Present Address:

M. Kyrish

Fresnel Technologies, 101 West Morningside Drive, Fort Worth, TX 76110, USA

A. P. Benveniste $\cdot$ W. Yang

Department of Diagnostic Radiology, University of Texas MD

Anderson Cancer Center, 1515 Holcombe Boulevard, Unit 1350,

Houston, TX 77030, USA of less than 2 min per core. Invasive tumor cellularity estimated from histologic and grayscale confocal images showed moderate agreement by kappa coefficient: $\kappa=0.48 \pm 0.09(p<0.001)$. Grayscale confocal images require less than $2 \mathrm{~min}$ for acquisition and allow for evaluation of invasive tumor cellularity in breast core needle biopsy specimens with moderate agreement to histologic images. We show that confocal fluorescence microscopy can be performed immediately following specimen acquisition and could indicate the need for additional biopsies at the initial visit.

Keywords Inflammatory breast cancer - Core needle biopsy · Confocal fluorescence microscopy $\cdot$ Tissue sampling

\section{Introduction}

Inflammatory breast carcinoma (IBC) is a rare, aggressive type of breast cancer that is characterized clinically by tenderness, erythema, and edema involving at least onethird of the entire breast [1-4]. This variant of breast cancer can be clinically mistaken for mastitis and treated with antibiotics resulting in a delay in the recognition of malignancy. The redness and edema of the breast is attributed to the frequent involvement of the lymphovascular channels in the breast. Pathologic diagnosis can be made using skin punch biopsy and/or core needle biopsy (CNB) of the affected breast. The presence of lymphovascular tumor emboli in the skin biopsy and invasive mammary carcinoma in the core biopsy can establish the diagnosis and initiate the clinical management of the patients. Tissue sampling can be a particularly problematic issue in IBC, since most cases present with no discrete 
mass and therefore accurate targeting of the area of breast for image-guided CNBs can be difficult $[5,6]$. Shah and colleagues showed that sampling error can lead to inadequate CNB specimens, which have an insufficient amount of tissue to make a diagnosis, in as many as $9.1 \%$ of cases [7]. In cases with sampling error, several more days are required to perform repeated biopsies and histologic assessment for additional tissue specimens [7, 8]. Immediate evaluation of CNBs for assessment of tumor cellularity can be extremely valuable to ensure procurement of adequate tissue not just for diagnosis of the invasive carcinoma but also for ancillary studies in one setting. Touch preparation and frozen section are the currently available techniques for rapid evaluation of breast CNB specimen adequacy. While touch preparation of breast CNBs can be useful to ascertain the presence or absence of tumor cells in the CNBs, it cannot indicate the extent of tumor cellularity in the CNB and whether the malignancy is in situ, invasive, or both. In addition, the process of touching and rolling the CNBs on the glass slides may cause distortion, which makes them less than optimal for accurate histopathological examination. Frozen section is generally not preferred for immediate assessment of CNBs because of loss of the precious tissue during the cutting process. A technique is needed to evaluate the adequacy of IBC CNB specimens as soon as they are procured in a clinical setting, without tissue preparation that may compromise the quality of the core. The technique should be able to immediately indicate to the radiologist if the area of interest has been accurately targeted and if additional biopsies are required [7].

Several studies have established the utility of confocal microscopy for breast tissue assessment [4, 9-12]. Schiffhauer and colleagues demonstrated the feasibility of confocal reflectance microscopy for identifying the presence of neoplasia in unfixed breast CNB specimens in real time [10]. We recently showed that confocal fluorescence microscopy can be performed in a clinical setting to acquire images of fresh breast tissue with sufficient resolution to identify neoplastic and non-neoplastic breast cytomorphological and architectural features [4].

The objective of this work was to determine the feasibility of using confocal fluorescence microscopy in a clinical setting to evaluate the adequacy of IBC CNB specimens by estimating the amount of invasive tumor cellularity in each specimen. To meet this objective, we performed a proof-of-concept study to estimate invasive tumor cellularity from grayscale confocal images and confocal images that were false-colored to mimic histologic staining; results were compared to the assessment from the gold standard of histologic images obtained from conventional light microscopic examination of formalinfixed and paraffin-embedded tissue sections of the core biopsies stained by hematoxylin and eosin.

\section{Materials and methods}

Core needle biopsy specimens were obtained from patients with clinical signs and symptoms of IBC. The protocol for tissue acquisition was reviewed and approved by the Institutional Review Boards at The University of Texas MD Anderson Cancer Center and Rice University. Ultrasound-guided CNBs were performed per standard clinical protocol. Single cores were prepared for confocal imaging within $5 \mathrm{~min}$ of the core needle biopsy procedure; each core was typically $1 \times 4 \times 12 \mathrm{~mm}^{3}$ in size. Cores were kept moist in phosphate buffered saline (PBS) (pH 7.4) prior to imaging. A solution of $0.01 \%$ proflavine in sterile PBS was applied topically to the core for approximately $1 \mathrm{~min}$; proflavine is a fluorescent dye that preferentially stains nuclei in confocal fluorescence images [13-16].

The procedure for imaging cores with confocal fluorescence microscopy has been described previously [4]. Prior to imaging, a core was positioned on the microscope stage. Each core was imaged using a scanning confocal microscope (Vivascope 2500 ${ }^{\circledR}$; Caliber Imaging and Diagnostics Inc., Rochester, New York) at $488 \mathrm{~nm}$ excitation with a $550 \pm 44 \mathrm{~nm}$ bandpass filter with a $30 \times$ water immersion lens (numerical aperture $=0.8$ ). At the center of the $750 \times 750 \mu \mathrm{m}^{2}$ region of interest (ROI), lateral resolution of the confocal microscope is $1.0 \mu \mathrm{m}$ and axial resolution is $5.0 \mu \mathrm{m}$. Initially, illumination power was set to $2.1 \mathrm{~mW}( \pm 0.4 \mathrm{~mW})$ and adjusted to maximize signal and avoid saturation. To create a composite image of the core at a single axial depth, the microscope stage was scanned in a grid pattern (maximum area: $12.2 \times 12.2 \mathrm{~mm}^{2}$ ); composite images of cores were typically $4 \times 12.2 \mathrm{~mm}^{2}$. After imaging, the cores were stored in PBS and submitted to the IBC tissue registry for processing, including standard histologic preparation, fixation, and staining with hematoxylin and eosin (H\&E).

Grayscale fluorescence images of cores were acquired with the confocal microscope. The grayscale confocal fluorescence images were false-colored to resemble histologic staining by combining the experimentally determined color values for hematoxylin and eosin reported by Gareau [17] into a single set of color values: $H=$ [1.3 0.751 .88 , where $[\operatorname{red}(k=1)$, green $(k=2)$, and blue $(k=3)]$. False color was applied to each pixel of confocal images $(C)$ as follows:

$C_{x, y, k}=1-F_{x, y}\left(2-H_{k}\right)$,

where $F$ is the grayscale confocal fluorescence image with each pixel normalized to the maximum [17]. The individual grayscale confocal images were automatically stitched together immediately following acquisition by the confocal microscope to form a composite grayscale image of each core. False-colored confocal images were manually 
Table 1 Percent invasive tumor cellularity in IBC cores estimated in histologic, grayscale confocal, and false-colored confocal images. Imaging time indicates the time to acquire a composite grayscale confocal image. The average imaging time was 01:54 mm:ss. Histologic IBC Type and Tumor Grade indicate the histologic type of inflammatory breast cancer and tumor grade, respectively, based on histologic assessment of all tissue specimens (including the core reviewed in this study) for each corresponding patient case

\begin{tabular}{|c|c|c|c|c|c|c|c|}
\hline \multirow[t]{2}{*}{$\begin{array}{l}\text { Adequacy for } \\
\text { tumor diagnosis }\end{array}$} & \multirow[t]{2}{*}{$\begin{array}{l}\text { Core } \\
\#\end{array}$} & \multicolumn{3}{|c|}{$\begin{array}{l}\text { Estimated invasive tumor cellularity in images } \\
\text { of core needle biopsy specimens }\end{array}$} & \multirow[t]{2}{*}{$\begin{array}{l}\text { Imaging time } \\
\text { (MM:SS) }\end{array}$} & \multirow[t]{2}{*}{ Histologic IBC type } & \multirow[t]{2}{*}{$\begin{array}{l}\text { Tumor } \\
\text { grade }\end{array}$} \\
\hline & & $\begin{array}{l}\text { Histology } \\
(\mathrm{H} \& \mathrm{E})\end{array}$ & $\begin{array}{l}\text { Confocal } \\
\text { (grayscale) }\end{array}$ & $\begin{array}{l}\text { Confocal (false } \\
\text { coloring) }\end{array}$ & & & \\
\hline \multirow[t]{20}{*}{ Adequate } & 1 & $90 \%$ & $75-80 \%$ & $80 \%$ & $00: 53$ & Invasive ductal carcinoma & 2 \\
\hline & 2 & $5 \%$ & $5 \%$ & $5 \%$ & $00: 53$ & $\begin{array}{l}\text { Invasive ductal carcinoma with } \\
\text { mucinous carcinoma }\end{array}$ & 3 \\
\hline & 3 & $10 \%$ & $25 \%$ & $20 \%$ & $02: 41$ & Invasive ductal carcinoma & 3 \\
\hline & 4 & $20 \%$ & $5 \%$ & $20 \%$ & 01:47 & Invasive ductal carcinoma & 2 \\
\hline & 5 & $75 \%$ & $75 \%$ & $20 \%$ & $02: 51$ & Invasive ductal carcinoma & 3 \\
\hline & 6 & $25 \%$ & $20 \%$ & $20 \%$ & 02:07 & Invasive ductal carcinoma & 3 \\
\hline & 7 & $20 \%$ & $20 \%$ & $20 \%$ & $02: 54$ & Invasive lobular carcinoma & 3 \\
\hline & 8 & $100 \%$ & $100 \%$ & $30 \%$ & $02: 51$ & Invasive ductal carcinoma & 3 \\
\hline & 9 & $5 \%$ & $5-10 \%$ & $5 \%$ & $02: 51$ & Invasive ductal carcinoma & 3 \\
\hline & 10 & $25-30 \%$ & $25 \%$ & $40 \%$ & 01:46 & Invasive lobular carcinoma & 2 \\
\hline & 11 & $95 \%$ & $80-95 \%$ & $90 \%$ & $02: 33$ & Invasive ductal carcinoma & 3 \\
\hline & 12 & $30 \%$ & $30 \%$ & $30 \%$ & 02:09 & Invasive ductal carcinoma & 3 \\
\hline & 13 & $30 \%$ & $20 \%$ & $50 \%$ & 01:01 & Invasive ductal carcinoma & 3 \\
\hline & 14 & $95 \%$ & $70 \%$ & $90 \%$ & 01:04 & Invasive ductal carcinoma & 3 \\
\hline & 15 & $80 \%$ & $75 \%$ & $90 \%$ & 01:04 & $\begin{array}{l}\text { Invasive ductal carcinoma with } \\
\text { micropapillary features }\end{array}$ & 2 \\
\hline & 16 & $70 \%$ & $90-95 \%$ & $95 \%$ & 01:04 & Invasive ductal carcinoma & 3 \\
\hline & 17 & $20 \%$ & $10 \%$ & $10 \%$ & 01:04 & $\begin{array}{l}\text { Invasive ductal and lobular } \\
\text { carcinoma }\end{array}$ & 1 \\
\hline & 18 & $50 \%$ & $10-15 \%$ & $15 \%$ & $02: 51$ & Invasive lobular carcinoma & 3 \\
\hline & 19 & $25 \%$ & $30 \%$ & $25 \%$ & 01:47 & Invasive ductal carcinoma & 2 \\
\hline & 20 & $30-40 \%$ & $40 \%$ & $20 \%$ & $01: 25$ & Invasive ductal carcinoma & 3 \\
\hline \multirow[t]{3}{*}{ Inadequate } & 21 & $0 \%$ & $0 \%$ & $5 \%$ & $02: 51$ & Invasive ductal carcinoma & 3 \\
\hline & 22 & $0 \%$ & $0 \%$ & $0 \%$ & 01:04 & Invasive ductal carcinoma & 3 \\
\hline & 23 & $0 \%$ & $0 \%$ & $0 \%$ & 01:47 & Invasive ductal carcinoma & 2 \\
\hline
\end{tabular}

$H \& E$ hematoxylin and eosin stain

stitched together, using the grayscale confocal image as a guide, to form a composite false-colored image of each core.

A board-certified, dedicated breast pathologist (author S. Krishnamurthy) assessed H\&E-stained slides with a $10 \times$ objective lens and evaluated confocal images at comparable magnification. Invasive tumor cellularity was estimated in three images for each core: one histologic image prepared with standard fixation in neutral buffered formalin, routinely processed and embedded in paraffin followed by H\&E staining, one composite grayscale confocal image, and one false-colored confocal image - using standard histologic criteria [18]. Tumor cellularity was defined as the percentage of the core comprised of invasive breast carcinoma [19].
A kappa coefficient was calculated for each combination of confocal and histologic image types (histologic vs. grayscale confocal images and histologic vs. false-colored confocal images), in order to compare the agreement between invasive tumor cellularity estimated from histologic, grayscale confocal, and false-colored confocal images. In this calculation of inter-rater agreement, each image type was considered to be one "rater."

\section{Results}

A total of 23 cores were collected from 23 patients with untreated, clinically suspected IBC [1] (Table 1). Twenty of the cores contained neoplastic tissue, identified by 
Fig. 1 Representative core with invasive tumor and nonneoplastic tissue (core 19); $25 \%$ cellularity estimated by author S.K. in histologic image (standard H\&E staining), $30 \%$ cellularity estimated in grayscale confocal fluorescence image $(0.01 \%$ proflavine staining), and $25 \%$ cellularity estimated in false-colored confocal fluorescence image derived from grayscale image (0.01\% proflavine staining). The solid outlined region indicates areas of the CNB specimen that contain invasive tumor. The scale bar at left is $750 \mu \mathrm{m}$. The insets at right show the border between neoplastic and non-neoplastic tissue in the CNB specimen. The locations of the insets are indicated in each core by a square with dashed lines. The scale bar at right is $100 \mu \mathrm{m}$
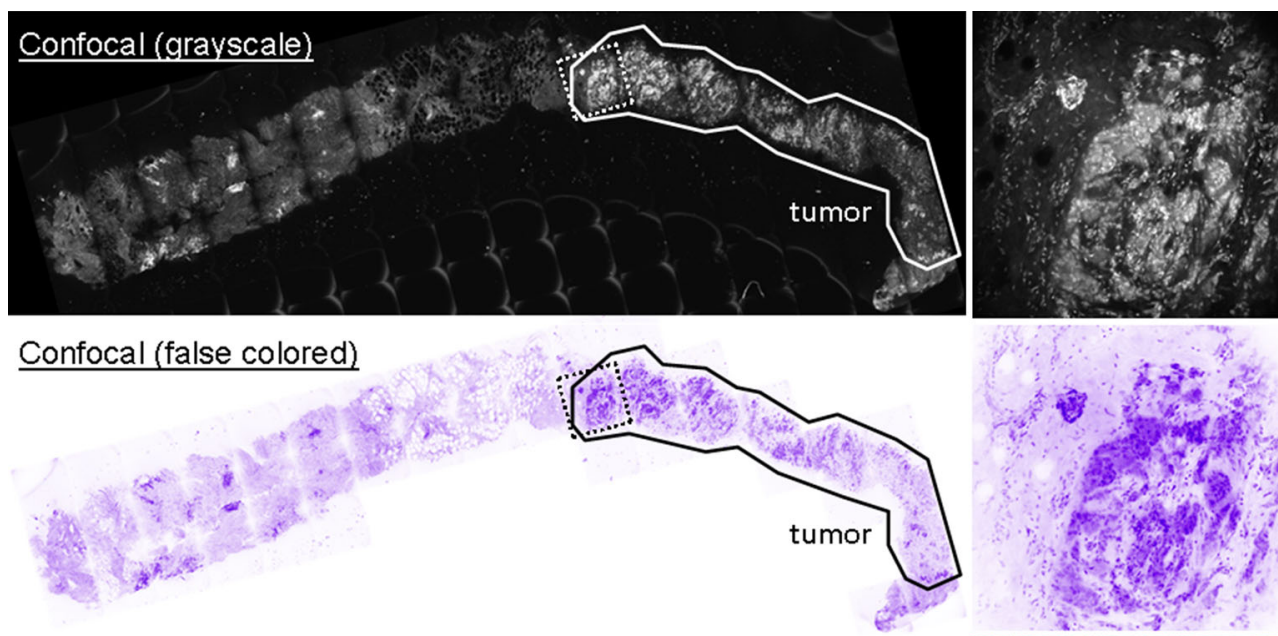

Histology (H\&E)
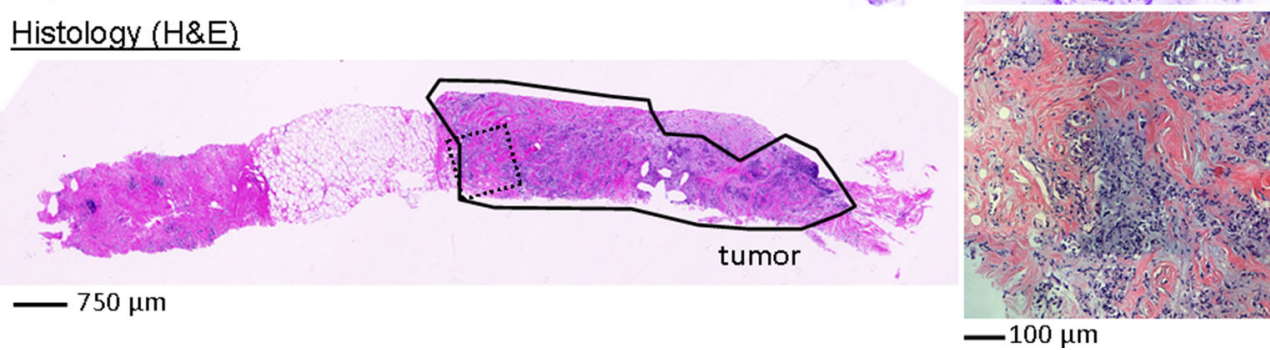

standard histologic criteria in histologic images. The average time to acquire a composite confocal image was $1 \mathrm{~min}, 54 \mathrm{~s}$ (Table 1). False coloring produced confocal images that visually resembled tissue sections stained with hematoxylin and eosin.

Representative histologic, grayscale confocal, and falsecolored confocal images of cores that contain IBC tissue are shown in Figs. 1 and 2. Figure 1 shows a core that contains both neoplastic and non-neoplastic tissue, with an estimated $25 \%$ cellularity in the histologic image; cellularity was estimated as 30 and $25 \%$ in grayscale confocal and false-colored confocal images, respectively. Figure 2 shows a core with $80 \%$ cellularity estimated in the histologic image, $75 \%$ cellularity estimated in the grayscale confocal image, and $90 \%$ cellularity estimated in the falsecolored confocal image.

Three cores acquired from patients diagnosed with IBC were considered to be inadequate as a result of sampling error; the histologic image contained no neoplastic tissue despite a clinical diagnosis of IBC. Representative images of an inadequate core consisting of adipose tissue and no neoplastic tissue, based on assessment of the histologic image, are shown in Fig. 3. All three images of the core estimated cellularity as $0 \%$.

Estimates of tumor cellularity based on histologic, grayscale confocal, and false-colored confocal images for all specimens are summarized in Table 1. Figure 4 illustrates the agreement between tumor cellularity estimated in histologic and grayscale confocal images (Fig. 4a) and in histologic and false-colored confocal images (Fig. 4b). Each data point represents a core needle biopsy specimen from which tumor cellularity was estimated. Dashed lines indicate the linear fit for each set of data points and Pearson coefficient $\left(R^{2}\right)$ values quantify the linear fit of the data. Tumor cellularity estimates were more consistent between histologic and grayscale confocal images $\left(R^{2}=0.88\right)$ than between histologic and false-colored confocal images $\left(R^{2}=0.61\right)$.

A kappa coefficient was calculated [20,21] to quantify the agreement between histologic images and either grayscale or false-colored images. Standard error and $p$ values for kappa were also quantified (Table 2). The kappa value calculated for agreement between histologic and grayscale confocal images indicates moderate agreement based on defined categories for kappa values [21, 22]; $\kappa=0.48 \pm 0.09(p<0.001)$. Agreement between histologic and false-colored confocal images was fair and not statistically significant; $\kappa=0.28 \pm 0.26(p=0.14)$.

\section{Discussion}

The objective of this work was to determine the feasibility of using confocal fluorescence microscopy for immediate assessment of invasive tumor cellularity of CNBs in patients with IBC. Composite grayscale confocal images of CNB specimens were acquired in an average time of $2 \mathrm{~min}$. Statistical analysis shows that there is a moderate 
Fig. 2 Representative core with invasive tumor (core 15); $80 \%$ cellularity estimated by author S.K. in histologic image (standard H\&E staining), $75 \%$ cellularity estimated in grayscale confocal fluorescence image $(0.01 \%$ proflavine staining), and $90 \%$ cellularity estimated in false-colored confocal fluorescence image derived from grayscale image (0.01\% proflavine staining). The solid outlined region indicates areas of the CNB specimen that contain neoplastic tissue. The scale bar at left is $750 \mu \mathrm{m}$. The insets at right show a region of neoplastic tissue in the CNB specimen. The locations of the insets are indicated in each core by a square with dashed lines. The scale bar at right is $100 \mu \mathrm{m}$

\section{Confocal (grayscale)}

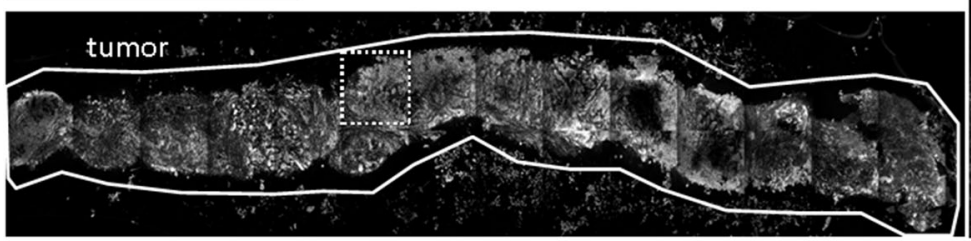

\section{Confocal (false colored)}

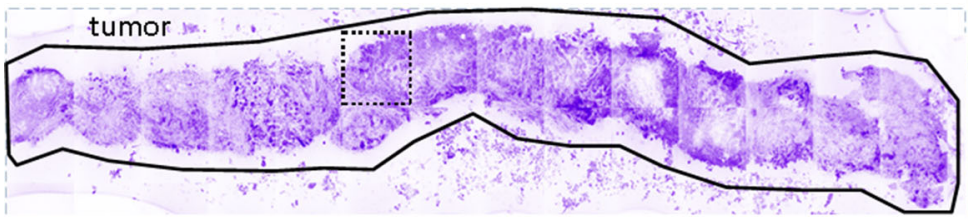

\section{Histology (H\&E)}

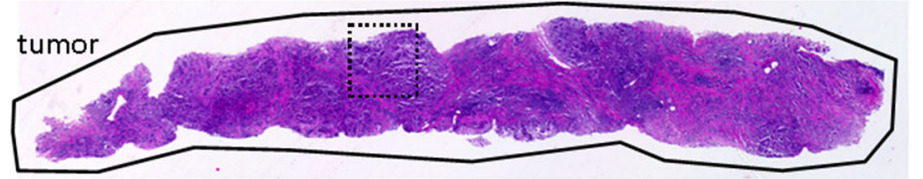

$750 \mu \mathrm{m}$
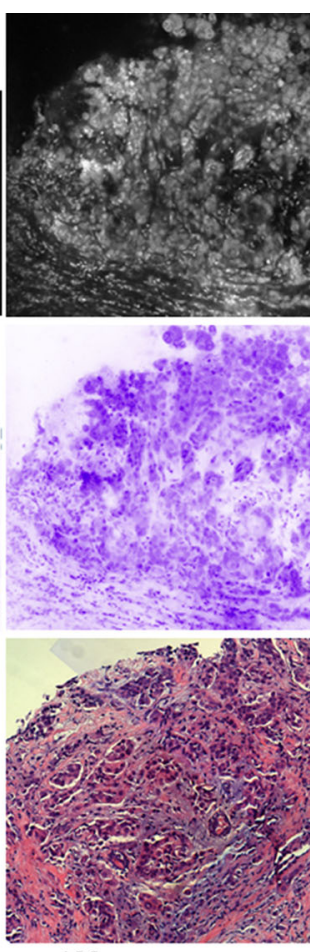

$-100 \mu \mathrm{m}$

Fig. 3 Representative core with no invasive tumor (core 22); $0 \%$ cellularity estimated by author S.K. in histologic image (standard H\&E staining), $0 \%$ cellularity estimated in grayscale confocal fluorescence image $(0.01 \%$ proflavine staining), and $0 \%$ cellularity estimated in false-colored confocal fluorescence image derived from grayscale image (0.01\% proflavine staining). This core is considered inadequate due to sampling error; no invasive tumor is visible despite a clinical indication of IBC. The scale bar at left is $750 \mu \mathrm{m}$. The insets at right show a region of nonneoplastic tissue in the $\mathrm{CNB}$ specimen. The locations of the insets are indicated in each core by a square with dashed lines. The scale bar at right is $100 \mu \mathrm{m}$

\section{Confocal (grayscale)}

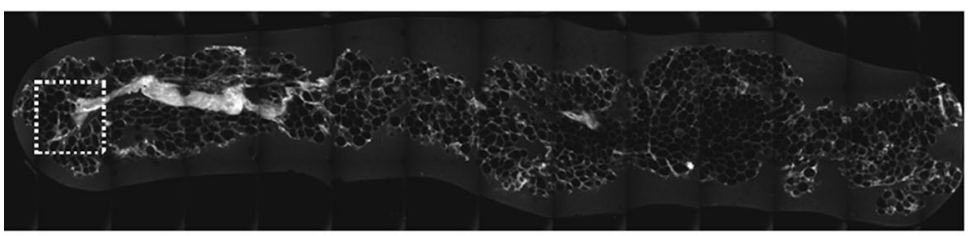

\section{Confocal (false colored)}
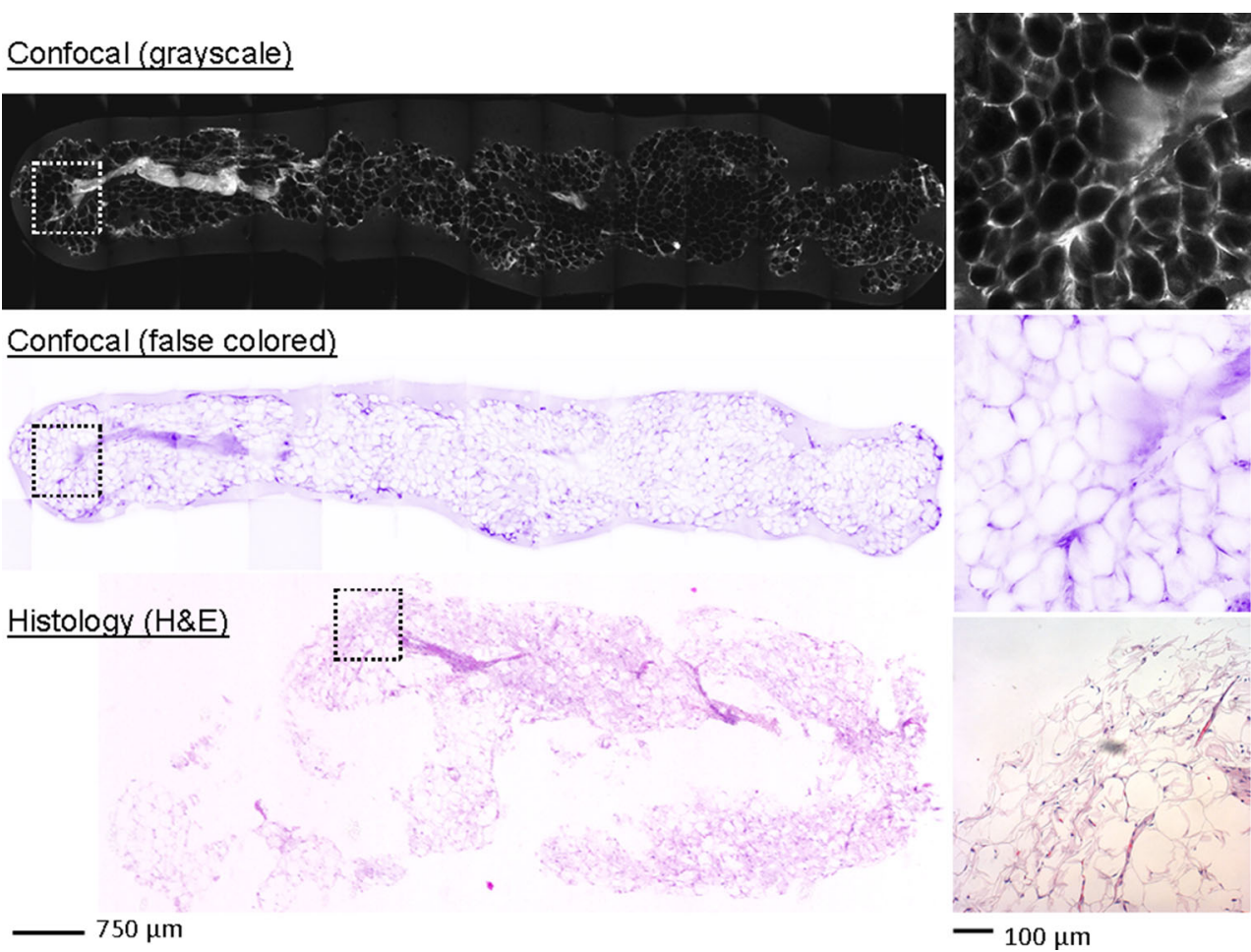

$-100 \mu \mathrm{m}$

agreement between tumor cellularity estimated from histologic images and grayscale confocal images. False-coloring the confocal images using a single analog for histologic staining does not improve agreement. These findings demonstrate that confocal fluorescence microscopy could be used to assess adequacy of IBC CNB specimens. This technique may be useful to ensure procurement of high-quality $\mathrm{CNBs}$ not only for routine 


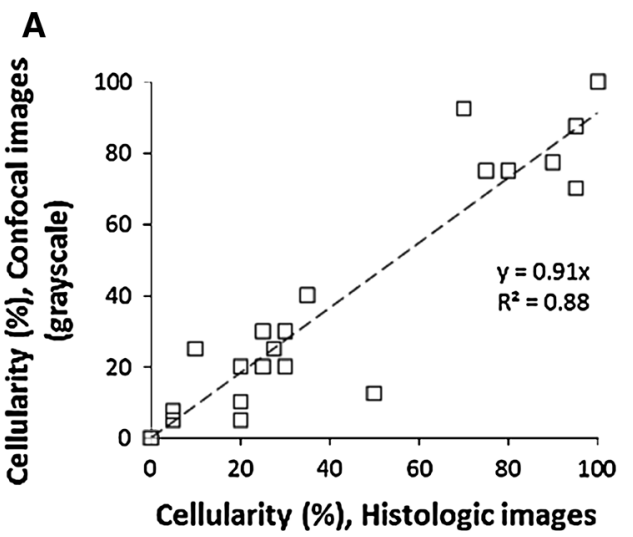

Fig. 4 Scatterplots illustrating agreement on estimated invasive tumor cellularity between image types. Each data point represents a CNB specimen. Dashed lines represent the linear fit of the data. Pearson coefficients $\left(R^{2}\right)$ quantify the linear fit of invasive tumor

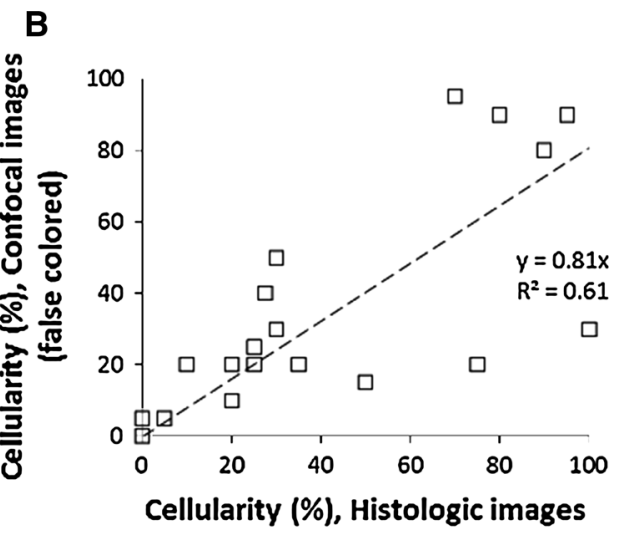

cellularity data. a Invasive tumor cellularity estimates from grayscale confocal images and histologic images. b Invasive tumor cellularity estimates from false-colored confocal images and histologic images

Table 2 Inter-rater agreement of estimated invasive tumor cellularity between the types of images, quantified by the kappa coefficient and standard error [20]. Level of agreement is based on categories described by Viera and Garrett [21]. $P$ value was calculated using a normal distribution

\begin{tabular}{|c|c|c|c|c|}
\hline \\
\hline \multicolumn{5}{|l|}{$\begin{array}{l}\text { Inter-rater agreement-two techniques } \\
\text { Image types }\end{array}$} \\
\hline Histologic versus grayscale confocal & 0.477 & 0.088 & Moderate & $3.43 \mathrm{E}-08$ \\
\hline Histologic versus false-colored confocal & 0.280 & 0.258 & Fair & 0.139 \\
\hline
\end{tabular}

histopathological diagnosis but also for ancillary studies including immunohistochemistry and molecular testing.

Of the three CNB specimens that were determined to be inadequate by histologic assessment, one had benign lobules and fibrous tissue (core \#21) and two consisted completely of adipose tissue (cores \#22, 23). These cores were identified as inadequate based on tumor cellularity estimated as $0-5 \%$ in histologic, grayscale confocal, and false-colored confocal images (Table 1). Of the $20 \mathrm{CNB}$ specimens that were determined to be adequate by histologic assessment, 20 were diagnosed as invasive carcinoma; morphological features of invasive carcinoma were also recognized in grayscale confocal and false-colored confocal images. One CNB specimen was diagnosed as mucinous carcinoma (core \#2) based on standard histologic criteria [18] and was also recognized as such on the confocal images.

IBC is an aggressive type of breast cancer, which is known to metastasize through the lymphatic system and typically presents as a non-palpable lesion [1-3]. To obtain a rapid and accurate diagnosis of $\mathrm{IBC}$, there must be a reliable method to sample tissue. Although inter-rater agreement is typically high for histologic diagnosis [2326], the rate of inadequate samples is a limitation of CNB as a sampling technique for breast cancer diagnosis [7, 8, $23,24,27]$. Shah and colleagues showed that the rate of sampling error leading to inadequate CNB specimens can be as high as $9.1 \%$ [7]. Therefore, there is a need for a technique that can evaluate specimen adequacy [7] with a relatively high sensitivity and specificity.

Previous studies have illustrated the feasibility of evaluating breast tissue specimens with confocal microscopy [4, 9-12]. Schiffhauer and colleagues evaluated confocal reflectance microscopy for screening unfixed breast CNB specimens with a range of neoplastic and non-neoplastic histologic features. Their study showed that there were adequate morphologic and cellular features in confocal reflectance images to allow correlation to histologic images [10]. In this study, we chose to focus on inflammatory breast cancer, because we wanted to determine if assessment of confocal fluorescence microscopy images of fresh cores would show which cores had insufficient tumor tissue for diagnosis and therefore identify cases for which a repeat biopsy was needed. Our previous work showed that confocal fluorescence microscopy images provide sufficient detail to distinguish between neoplastic breast features, such as ductal carcinoma in situ and invasive ductal and lobular carcinoma, and non-neoplastic breast features including benign ducts, lobules, and hyperplasia. [4] We characterized inter-rater agreement between pathologists who assessed confocal fluorescence with a kappa coefficient for multiple ratings per subject. The kappa coefficient 
described in our previous work $(0.51, p<0.001)$ between pathologists assessing confocal images indicated moderate agreement based on defined categories of kappa coefficients $[21,22]$. This level of agreement is similar to the moderate agreement between a single rater's assessment of grayscale confocal and histologic images shown in Table 2 $(0.477, p<0.001)$. The findings of these two studies suggest that confocal fluorescence images could potentially be used to assess adequacy of breast core needle biopsies with many histologic features.

False coloring to mimic histologic staining in confocal images of breast tissue has been demonstrated in two studies $[17,28]$. Gareau showed that the false-coloring technique using confocal reflectance and fluorescence microscopy to mimic hematoxylin and eosin staining, respectively, produced multimodal confocal images mimicking the appearance of corresponding histologic images [17]. Bini and colleagues used false coloring for tissue obtained from Mohs skin excisions and demonstrated correlation between confocal mosaics and histologic images [28]. Previous studies showed that false coloring improved correlation between confocal and histologic images [21, 22]. However, in contrast to the previous studies which used both reflectance and fluorescence images to mimic hematoxylin and eosin staining, we used only fluorescence images to mimic histologic staining of the tissue section. It was not possible to use multimodal confocal images because reflectance images were not collected for every CNB specimen and because some confocal reflectance images were not aligned laterally or axially with the confocal fluorescence images of the corresponding core needle biopsy specimen.

In conclusion, we present the results of a proof-ofconcept study comparing estimated invasive tumor cellularity between grayscale confocal, false-colored confocal, and histologic images of 23 IBC CNB specimens. Grayscale confocal images require an average of only $2 \mathrm{~min}$ for acquisition and allow immediate evaluation of invasive tumor cellularity in IBC CNB specimens; agreement with histologic images is moderate. We show that confocal fluorescence microscopy could be used for immediate assessment of CNB specimen adequacy, so that additional biopsies for diagnosis, bio-banking, or genetic sequencing studies can be performed on site without need for a repeat visit. Additional larger prospective studies are warranted to validate our findings for the potential utilization of confocal fluorescence microscopy in routine pathology practice as a robust technique for immediate assessment of specimen adequacy of IBC CNBs.

Acknowledgments The authors would like to thank Dongsuk Shin for his assistance with the code for false-coloring confocal fluorescence images, and acknowledge support from the Department of
Defense Era of Hope Award W81XWH-09-1-0410, and the Susan G. Komen for the Cure grant KG091020. This work was also supported in part by The State of Texas Grant for Rare and Aggressive Cancers through the Morgan Welch Inflammatory Breast Cancer Research Program.

Conflicts of interest The authors declare that they have no conflicts of interest.

Open Access This article is distributed under the terms of the Creative Commons Attribution Noncommercial License which permits any noncommercial use, distribution, and reproduction in any medium, provided the original author(s) and the source are credited.

\section{References}

1. Yamauchi H, Woodward WA, Valero V, Alvarez RH, Lucci A, Buchholz TA et al (2012) Inflammatory breast cancer: what we know and what we need to learn. Oncologist 17:891-899

2. Hance KW, Anderson WF, Devesa SS, Young HA, Levine PH (2005) Trends in inflammatory breast carcinoma incidence and survival: the surveillance, epidemiology, and end results program at the National Cancer Institute. J Natl Cancer Inst 97:966-975

3. Anderson WF, Chu KC, Chang S (2003) Inflammatory breast carcinoma and noninflammatory locally advanced breast carcinoma: distinct clinicopathologic entities? J Clin Oncol 21:2254-2259

4. Dobbs JL, Ding H, Benveniste AP, Kuerer HM, Krishnamurthy S, Yang W, Richards-Kortum R (2013) Feasibility of confocal fluorescence microscopy for real-time evaluation of neoplasia in fresh human breast tissue. J Biomed Opt. doi:10.1117/1.JBO.18. 10.106016

5. Kumar N, Sayed S, Moloo Z, Wasike R (2011) Fine-needle aspiration in suspected inflammatory breast cancer: case series with emphasis on approach to specimen adequacy. Acta Cytol 55:239-244

6. Iwase T, Takahashi K, Gomi N, Horii R, Akiyama F (2006) Present state of and problems with core needle biopsy for nonpalpable breast lesions. Breast Cancer 13:32-37 Tokyo, Japan

7. Shah VI, Raju U, Chitale D, Deshpande V, Gregory N, Strand V (2003) False-negative core needle biopsies of the breast-an analysis of clinical, radiologic, and pathologic findings in 27 consecutive cases of missed breast cancer. Cancer 97:1824-1831

8. Dillon MF, Hill ADK, Quinn CM, O'Doherty A, McDermott EW, O'Higgins N (2005) The accuracy of ultrasound, stereotactic, and clinical core biopsies in the diagnosis of breast cancer, with an analysis of false-negative cases. Ann Surg 242:701-707

9. Parrish A, Halama E, Tilli MT, Freedman M, Furth PA (2005) Reflectance confocal microscopy for characterization of mammary ductal structures and development of neoplasia in genetically engineered mouse models of breast cancer. J Biomed Opt. doi: $10.1117 / 1.2065827$

10. Schiffhauer LM, Boger JN, Bonfiglio TA, Zavislan JM, Zuley M, Fox CA (2009) Confocal Microscopy of Unfixed Breast Needle Core Biopsies: A Comparison to Fixed and Stained Sections. BMC Cancer. doi: 10.1186/1471-2407-9-265

11. Tilli MT, Cabrera MC, Parrish AR, Torre KM, Sidawy MK, Gallagher AL, et al (2007) Real-time imaging and characterization of human breast tissue by reflectance confocal microscopy. J Biomed Opt. doi: 10.1117/1.2799187

12. Abeytunge S, Li Y, Larson B, Peterson G, Seltzer E, ToledoCrow R, et al (2013) Confocal microscopy with strip mosaicing for rapid imaging over large areas of excised tissue. J Biomed Opt. doi: 10.1117/1.JBO.18.6.061227 
13. Muldoon TJ, Anandasabapathy S, Maru D, Richards-Kortum R (2008) High-resolution imaging in Barrett's esophagus: a novel, low-cost endoscopic microscope. Gastrointest Endosc 68:737-744

14. Muldoon TJ, Pierce MC, Nida DL, Williams MD, Gillenwater A, Richards-Kortum R (2007) Subcellular-resolution molecular imaging within living tissue by fiber microendoscopy. Opt Express 15:16413-16423

15. Rosbach KJ, Shin D, Muldoon TJ, Quraishi MA, Middleton LP, Hunt KK et al (2010) High-resolution fiber optic microscopy with fluorescent contrast enhancement for the identification of axillary lymph node metastases in breast cancer: a pilot study. Biomed Opt Express 1:911-922

16. Pierce MC, Javier DJ, Richards-Kortum R (2008) Optical contrast agents and imaging systems for detection and diagnosis of cancer. Int J Cancer 123:1979-1990

17. Gareau DS (2009) Feasibility of digitally stained multimodal confocal mosaics to simulate histopathology. J Biomed Opt. doi: $10.1117 / 1.3149853$

18. Rosen PP (2008) Rosen's breast pathology, 3rd edn. Lippincott Williams \& Wilkins, Philadelphia

19. Rajan R, Poniecka A, Smith TL, Yang Y, Frye D, Pusztai L et al (2004) Change in tumor cellularity of breast carcinoma after neoadjuvant chemotherapy as a variable in the pathologic assessment of response. Cancer 100:1365-1373

20. Fleiss JL, Levin B, Paik MC (2003) The measurement of interrater agreement. Statistical methods for rates and proportions, 3rd edn. Wiley, Hoboken
21. Viera AJ, Garrett JM (2005) Understanding interobserver agreement: the kappa statistic. Fam Med 37:360-363

22. Landis JR, Koch GG (1977) The measurement of observer agreement for categorical data. Biometrics 33:159-174

23. Perrot N, Jalaguier-Coudray A, Frey I, Thomassin-Naggara I, Chopier J (2013) US-guided core needle biopsy: false-negatives. How to reduce them? Eur J Radiol 82:424-426

24. Youk JH, Kim E-K, Kim MJ, Kwak JY, Son EJ (2010) Analysis of false-negative results after US-guided 14-gauge core needle breast biopsy. Eur Radiol 20:782-789

25. Garg S, Mohan H, Bal A, Attri AK, Kochhar S (2007) A comparative analysis of core needle biopsy and fine-needle aspiration cytology in the evaluation of palpable mammographically suspicious breast detected lesions. Diagn Cytopathol 35:681-689

26. Collins LC, Connolly JL, Page DL, Goulart RA, Pisano ED, Fajardo LL et al (2004) Diagnostic agreement in the evaluation of image-guided breast core needle biopsies - Results from a randomized clinical. Am J Surg Pathol 28:126-131

27. Peter D, Gruenhagen J, Wenke R, Schaefer FKW, Schreer I (2008) False-negative results after stereotactically guided vacuum biopsy. Eur Radiol 18:177-182

28. Bini J, Spain J, Nehal K, Hazelwood V, DiMarzio C, Rajadhyaksha M (2011) Confocal mosaicing microscopy of human skin ex vivo: spectral analysis for digital staining to simulate histology-like appearance. J Biomed Opt. doi: 10.1117/1.3596742 